\title{
XXXIV. The oscillatory discharge of a large accumulator
}

\author{
John Trowbridge
}

To cite this article: John Trowbridge (1897) XXXIV. The oscillatory discharge of a large accumulator, Philosophical Magazine Series 5, 44:268, 259-262, DOI: 10.1080/14786449708621061

To link to this article: http://dx.doi.org/10.1080/14786449708621061

曲 Published online: 08 May 2009.

Submit your article to this journal $[\pi$

Џ Article views: 2

Q View related articles $\asymp$ 
Supposing that the apparatus was arranged in a horizontal plane, then the reflexions (under $45^{\circ}$ ) from the two plane plates, one unsilvered and one semisilvered with a transparent film of silver, must weaken especially the horizontal vibrations and hence almost annihilate their influence. For unsilvered plates it is easily calculated that the intensity of horizontal vibrations is under the circumstances stated weakened five or six times more than that of the vertical ones. This ratio is of course somewhat changed by the silver film. How much cannot be said without knowledge of further details.

If I have indicated the real cause Michelson has observed a case analogous to my triplet $a$, when a nicol is interposed in such a position as to get rid of the horizontal vibrations. Let us hope that Prof. Michelson will soon give us his opinion.

Amsterdam, July 10.

[Note added August 10.]-Prof. Michelson kindly informs me just now that he believes my explanation of the discrepancy in our results to be correct.

XXXIV. The Oscillatory Discharge of a Large Accumulator. By JoHN TrowbrIDGE*

$\mathrm{T}$ HE discharge from a large number of Planté cells is characterized by a sibilant flame which, by quickly separating the spark-terminals, can be drawn out to a length of several feet. It closely resembles the light produced by passing an electric spark through lycopodium powder. When a photograph of this flaming discharge is examined it is seen to have an intensely bright spark as a nucleus (fig. 1). On account of the flaming discharge it is difficult to examine its character by means of a revolving mirror. By employing, however, two spark-gaps it seemed possible to ascertain whether the discharge is oscillatory or not.

In my experiments the circuit was made at the instant the revolving mirror was in the position to reflect an image of the discharge of the battery upon a sensitive plate. The photographs obtained in this way showed disruptive discharges superimposed upon continuous discharge. The latter, however, masked any appearance of an oscillatory discharge. It was evidently necessary to blow out the flaming discharge in order to see if oscillations followed the pilot discharge. The first experiment was made with 2500 cells arranged in series ; and the flaming discharge was much lessened both by the reduction in the number of cells and by a suitable arrange-

* Communicated by the Author. 
ment for blowing it out. On developing the photographs it was found that the discharge was an oscillatory one, for as many as five or six clearly defined oscillations followed the first, or pilot discharge. The number of cells was then doubled, and, although more difficulty was experienced with the flaming discharge, oscillations were again obtained.

Fig. 1.

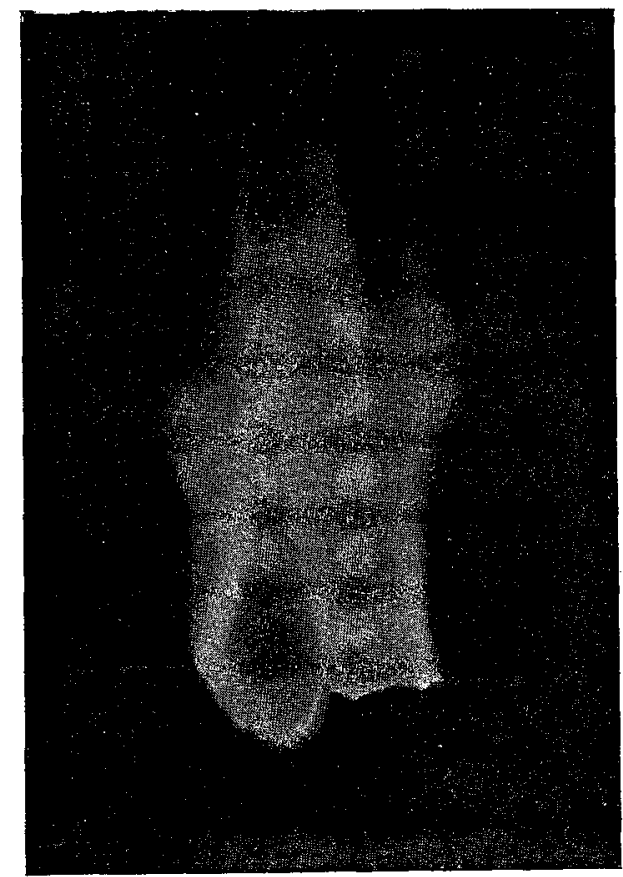

On the supposition that each cell of the battery can be regarded as a leaking condenser, and that it is equivalent in capacity to a condenser shunted by a resistance equal to that of the electrolyte, we can treat such a cell as a conducting condenser under the influence during discharge of a periodic current. The analysis of the well-known case is as follows:Let $A B C$ and $A E C$ be two circuits*, the current $A B C$ being a shunt to the circuit AEC which contains a condenser $\mathrm{E}$. Let $\mathrm{L}$ be the coefficient of self-induction of $\mathrm{ABC}$, $R$ its resistance, $C$ the capacity of the condenser in the current $\mathrm{AEC}$, and $r$ the resistance of the wires leading to the

* 'Elements of Electricity and Magnetism,' Prof. J. J. Thomson, p. 431. 
plates of the condenser. Then if $i$ is the current through $\mathrm{ABC}$, and $x$ the charge on the plate nearest to $\mathrm{A}$,

$$
\mathrm{L} \frac{d i}{d t}+\mathrm{R} i=r \frac{d x}{d t}+\frac{x}{c},
$$

since each of the quantities is equal to the electromotive force between $A$ and $C$.

If $i=\cos p t$, then

$$
x=\frac{\left(\mathrm{L}^{2} p^{2}+\mathrm{R}^{2}\right)^{\frac{1}{2}}}{\left(\frac{1}{\mathrm{C}^{2}}+r^{2} p^{2}\right)^{\frac{1}{9}}} \sin (p t+\mathrm{A}),
$$

where

$$
\mathrm{A}=\tan ^{-1} \frac{\mathrm{L} p}{\mathrm{R}}+\tan ^{-1} \frac{1}{r p c} \text {. }
$$

Hence

$$
-\frac{d x}{d t}=\sqrt{\frac{\overline{\mathrm{L}^{2} p^{2}+\mathrm{R}^{2}}}{\frac{1}{\mathrm{C}^{2} p^{2}}+r^{2}}} \cos (p t+\mathrm{A}) .
$$

Thus the maximum current along $\mathrm{AEC}$ is to that along $\mathrm{ABC}$ as $\sqrt{\mathrm{L}^{2} p^{2}+\mathrm{R}^{2}}$ is to $\sqrt{\frac{1}{\mathrm{C}^{2} p^{2}}+r^{2}}$; or if we neglect the resistance $r$ of the leading wires, as $\sqrt{\mathrm{L}^{2} p^{2}+\mathrm{R}^{2}}: \frac{1}{\mathrm{CP}}$; or neglecting $\mathrm{L}$, as $\frac{\mathrm{R}}{\frac{1}{\mathrm{C} p}}$.

In the case of one cell of the battery the polarizationcapacity is undoubtedly very large. G. M. Gordon* finds that the polarization-capacity of the surfaces of platinum $0.65 \mathrm{~cm} .^{2}$, separated by an interval of $2 \mathrm{~mm}$., amounts to more than 50 microfarads. The cells of my battery consist of lead plates of about $10 \mathrm{~cm}^{2}$. surface separated by about $6 \mathrm{~mm}$. The layer of peroxide of lead undoubtedly gives a very large polarization-capacity. The resistance of each cell is about one quarter of an ohm. Even with the small value of $\mathrm{R}$, under the effect of rapidly oscillating currents, such as my experiments show arise when the battery discharges through air or gases, a large portion of the oscillating currents pass through the condenser-circuit. Since the electrolyte acts as a semi-insulator, with a very high value of $p$, no current would pass through the condenser-circuit and the electrolyte, and the cells would therefore act like leyden jars.

* Wied. Ann. No. 5, 1897, p. 28.

Phil. Mag. S. 5. Vol. 44. No. 268. Sept. 1897. 
In the case I am considering the Planté cells evidently act like leaky leyden jars coupled in series. If $\mathrm{C}$ is the apparent capacity of one cell, $\frac{\mathrm{C}}{n}$ would be the capacity of $n$ cells.

An examination of the photographs of the oscillations produced by 2500 cells showed an apparent capacity of about 1000 electrostatic units. Five thousand cells gave an apparent capacity of about 500 units. The small apparent capacity $\mathrm{C}$ results from the leaking of the condenser due to the conduction through the electrolyte. Since the discharge from an accumulator of a large number of cells is in general oscillatory, I am led to the belief that the discharge from any primary battery is also oscillatory for all cases in which we have to deal with capacity and self-induction. It is evident that a galvanometer or telephone in circuit with a Geissler tube cannot detect the oscillatory discharge, since it is of high period. Moreover, when a Geissler tube is lighted by a large battery with no resistance save that of the tube and the battery in the circuit, and the light is examined in a revolving mirror by the eye, no oscillation or intermittence of light can be perceived on account of the flaming discharge through the rarefied gas.

The oscillatory discharge may be said to be the common occurrence in nature in the case of electrical discharges, and the one-direction discharge the uncommon. This has been expressed by the remark that electricity takes the path of least resistance, this common belief must be modified, however, under certain conditions of resonance; but in general nature avoids a unidirectional discharge.

Jefferson Physical Laboratory,

Harvard University,

Cambridge, Mass., U.S.

XXXV. A Method of determining Magnetic Hysteresis loss in Straight Iron Strips. By J. A. F́leming, M.A., D.Sc., $F^{T}$.R.S., Professor of Electrical Engineening in University College, London *.

[Plate V.]

THE exact determination of the magnetic bysteresis loss 1 in sheet iron as used for alternating current transformer construction has become of commercial importance of late years. Owing to the obvious necessity for reducing the core loss in transformers to the lowest attainable limit, it is now the custom to carefully test the iron to be used and to express

* Comnunicated by the Physical Society : rerd June 11th, 1897. 